\title{
Breite Ablehnung der ambulanten Kodierrichtlinien
}

- Auch die Vertreterversammlung der KV Niedersachsen (KVN) hat sich in der letzten Sitzung ihrer Wahlperiode klar gegen die Einführung der neuen Ambulanten Kodierrichtlinien (AKR) ausgesprochen. Sie fordert deren Aussetzung zum jetzigen Zeitpunkt und im jetzigen Um- fang und vor einer Umsetzung zunächst weitere Erprobungen, da erst nach deren Bewertung über die Einführung erneut entschieden werden könne. Es müsse sichergestellt sein, dass es zu keinen zusätzlichen bürokratischen Belastungen der Ärzteschaft kommt.

\author{
„Durch die in den AKR praktizierte \\ enorme Kodiertiefe dürften das \\ traditionell gute Arzt-Patienten-Verhältnis \\ und das Patientengeheimnis erheblich \\ gestört werden."
}

\section{AKR als Munition für Versicherungen}

\section{Grundsätzlich kein Vertragsabschluss}

(auch keine Lebens- oder Rentenversicherung)

- Alkoholiker

- Drogenabhängige

- Menschen aus dem Rotlichtmilieu

\section{Im Regelfall kein Vertragsabschluss}

(Ausnahmen im begründeten Einzelfall möglich)

- Geistes- oder Gemütserkrankungen

- Psychosomatische Erkrankungen

- Mobbingopfer

_ Multiple Sklerose

- Krebs

_ Diabetes mellitus

\section{Vertragsabschluss nur mit Ausschluss möglich}

- Erkrankungen des Stütz- und Bewegungsapparates

- Hexenschuss, Lumbalgie, HWS-, BWS- und LWS-Syndrom

- Bandscheibenvorfall

- Meniskusoperation

- Tennisarm etc.

- Allergien (z. B. Heuschnupfen, Nickelallergie)

- Bronchialasthma

- Migräne

\section{Versicherung nur mit Risikozuschlag \\ - Kreislauferkrankungen (z. B. Bluthochdruck) \\ - Erkrankungen der Verdauungsorgane \\ - Stoffwechselstörungen (z. B. erhöhte Cholesterinwerte)}

Eine solche Risikobewertungsliste legen die meisten Versicherungsgesellschaften im Rahmen eines beantragten Versicherungsschutzes zugrunde. Welche Chancen ein Patient nach AKR-Einführung nach einem Arztbesuch noch hat, ohne Zuschläge auszukommen, lässt sich ohne weitere Kommentare erkennen!

\section{MMW Kommentar}

Bereits die Vertreterversammlungen in Nordrhein, Bayern, Schleswig-Holstein, Hamburg, Westfalen-Lippe und Hessen sowie verschiedene Berufsverbände und Ärzteorganisationen hatten zuvor die Einführung der ambulanten Kodierrichtlinien als eine überzogene $\mathrm{Ma} \beta$ nahme angesehen, die nicht dazu geeignet ist, die Morbidität der Patienten besser abzubilden, als dies unter der jetzigen Anwendung der ICD-10-Kodierung bereits möglich ist.

Nach Feldversuchen in Hessen in ausgewählten Praxen auf freiwilliger Basis konnte darüber hinaus bewiesen werden, dass eine Umsetzung der jetzt vorgelegten AKR in einer durchschnittlich großen hausärztlichen Praxis zu einer täglichen Mehrbelastung von ein bis zwei Stunden und im Rahmen der Abrechnungserstellung zu einer notwendigen Praxisschließung von bis zu einer Woche führt. Hinzu kommt, dass durch die in den AKR praktizierte enorme Kodiertiefe das traditionell gute Arzt-Patienten-Verhältnis und das Patientengeheimnis erheblich gestört werden dürfte.

Wer künftig auch nur wegen einer Verspannung im HWS-Bereich oder eines saisonalen Heuschnupfens die Praxis aufsucht, muss nämlich damit rechnen, will er irgendwann eine Lebens- oder Unfallversicherung abschließen oder in die private Krankenversicherung (PKV) wechseln, dass dies entweder überhaupt nicht oder nur mit erheblichen Beitragszuschlägen möglich ist. Der Arzt muss die verordneten Arznei- oder Heilmittel nämlich nach AKR kodieren, sonst werden seine abgerechneten Leistungen nicht akzeptiert und die gesamte Quartalsabrechnung kann nicht erstellt werden. Fragt später eine Versicherungsgesellschaft unter Hinweis auf die Befreiung von der Verschwiegenheitsverpflichtung durch den Patienten nach diesen Behandlungsdaten, könnte es sogar noch zu nachträglichen Diskussionen mit dem Patienten kommen, weil der diese Zusammenhänge nicht kennt. 